\title{
Biodemas de cepas do Trypanosoma cruzi isoladas de humanos de trêsáreas endêmicas de Minas Gerais
}

\author{
Biodemes of Trypanosoma cruzi strains isolated from humans \\ from three endemic areas in Minas Gerais State

\begin{abstract}
Rodolfo Devera ${ }^{1,4}$, Ximena Illarramendi', Roberto Montoya-Araújo', Claude Pirmez ${ }^{2}$, Octavio Fernandes ${ }^{1,3}$ e José Rodrigues Coura ${ }^{1}$
\end{abstract}

\begin{abstract}
Resumo Com o objetivo de estudar o comportamento biológico de cepas do T. cruzi e determinar a possível relação entre o biodema da cepa e as formas clínicas da doença de Chagas, 14 cepas do protozoário isoladas de humanos, procedentes de Pains, Iguatama e Berilo, foram avaliadas. Para estudar o comportamento biológico das cepas, grupos de camundongos albinos suíços, machos, pesando entre 10-15 gramas foram infectados com $1 \times 10^{4}$ tripomastigotas sangüíneos para avaliação da infectividade, parasitemia, morfologia dos tripomastigotas sangüíneos, virulência e distribuição do protozoário nos tecidos. Nove cepas apresentaram comportamento similar ao da cepa São Felipe do biodema Il e cinco se caracterizaram como pertencentes ao biodema III. Não foi possível estabelecer uma relação entre o biodema da cepa e gravidade da doença nos pacientes.
\end{abstract}

Palavras-chaves: Trypanosoma cruzi. Biodema. Comportamento biológico. Doença de Chagas.

\begin{abstract}
In order to study the biological behavior of T. cruzi strains and to determine a putative association between their biodeme and the clinical forms of Chagas disease, 14 strains isolated from humans were evaluated. The individuals were from the municipalities of Pains, Iguatama and Berilo (Minas Gerais State). The biological behavior was evaluated in albino swiss mice, weighing 10 to $15 \mathrm{grams}$, which were infected with $1 \times 10^{4}$ blood tripomastigotes. The infectivity, parasitemia, tripomastigote morphology, virulence and the tissue distribution of the protozoan were analyzed. A behavior similar to biodeme II (São Felipe strain) was observed in 9 strains, while 5 stocks were characterized as belonging to biodeme III. It was not possible to establish a relationship between the biodeme strain and the severity of the disease in the patients.
\end{abstract}

Key-words: Trypanosoma cruzi. Biodeme. Biological behaviour. Chagas' disease.

Trypanosoma cruzi é um protozoário hemoflagelado causador da doença de Chagas (DC) que, segundo estimativas da OMS, afeta 18 milhões de pessoas na América Latina ${ }^{21}$. A doença se caracteriza pela sua variedade nas apresentações clínicas que incluem a forma indeterminada, cardiomiopatia e alterações digestivas ${ }^{15}$. Variações intraespecificas nas diferentes cepas do T. cruziestudadas têm sido demonstradas tanto em nível morfo-biológico, bioquímico e genético 126101118 19. Essa heterogeneidade poderia ser uma das razões que expliquem a variabilidade nas manifestações clinicas da DC e as diferenças regionais de sua morbidade.
Estudos sobre o comportamento biológico de cepas do $T$. cruzi e seus perfis histopatológicos em animais experimentais têm permitido agrupar os isolados em três tipos ou biodemas: I, II e III que por sua vez correspondem a zimodemas específicos. O biodema I, cujo protótipo é a cepa Y, é constituído por cepas com predomínio de formas delgadas e macrofagotropismo na fase inicial da infecção. Se multiplicam rapidamente, apresentando elevada parasitemia e mortalidade dos camundongos, que evoluem para o óbito entre o 7 e 12 dias após a inoculação. O biodema II que possui como protótipo a cepa São Felipe, se revela com

\footnotetext{
1. Departamento de Medicina Tropical do Instituto Oswaldo Cruz da Fundação Oswaldo Cruz, Rio de Janeiro, RJ. 2. Departamento de Bioquímica e Biologia Molecular do Instituto Oswaldo Cruz da Fundação Oswaldo Cruz, Rio de Janeiro, RJ. 3. Departamento de Patologia da Universidade do Estado do Rio de Janeiro, RJ. 4. Departamento de Parasitología y Microbiología da Escuela de Medicina da Universidad de Oriente, Ciudad Bolívar, Venezuela. Apoio financeiro: Conselho Nacional de Desenvolvimento Científico e Tecnológico-CNPq-Brasil e FIOCRUZ.

Endereço para correspondência: Dr. Rodolfo Devera. Dept ${ }^{\circ}$ de Medicina Tropical/IOC/FIOCRUZ, 21045-900 Rio de Janeiro, RJ.

Telefax: $55212280-3740$.

e-mail: rdevera@gene.dbbm.fiocruz.br

Recebido para publicação em 19/6/2001.
} 
predominância de formas largas, miocardiotropismo, multiplicação relativamente lenta e picos de parasitemia irregulares entre os 12 e 20 dias após a infecção, período no qual também a mortalidade é máxima. O biodema III, representado pela cepa Colombiana, é composto por cepas com predomínio de formas largas, baixa multiplicação, picos de parasitemia tardios entre os dias 20 e 30 após a infecção; acometimento principal da musculatura esquelética e com baixa taxa de tardia mortalidade (50 dias após a infecção) ${ }^{123}$.

Em uma determinada área geográfica pode estar circulando um ou vários padrões de cepas ou biodemas do T. cruzi, porém geralmente com predomínio de um deles ${ }^{13}$.
Uma cepa do T. cruzi pode estar formada por diferentes populações, com predominância de clones principais que apresentam um comportamento biológico particular ${ }^{19} 20$. A distribuição dos diferentes biodemas em diversas áreas endêmicas é importante para estabelecer a sua possível influência nas manifestações regionais da $\mathrm{DC}^{3}$.

Os objetivos do presente trabalho foram: 1) estudar o comportamento biológico de cepas do T. cruzi isoladas de pacientes chagásicos crônicos procedentes de três áreas endêmicas de Minas Gerais, para determinar o biodema predominante em cada uma das áreas estudadas; 2) determinar a relação entre o biodema da cepa e as manifestações clínicas locais da DC.

\section{MATERIAL E MÉTODOS}

Pacientes e cepas do T. cruzi. Quatorze pacientes sorologicamente positivos (Imunofluorescênica indireta e ELISA) foram submetidos a xenodiagnóstico com 20 ninfas de quarto estágio de Triatoma infestans e igual número de Panstrongylus megistus. Nove pacientes eram procedentes do município de Berilo (nordeste de Minas Gerais), 2 indivíduos viviam no município de Pains e 3 no município de Iguatama (sudeste de Minas Gerais). As avaliações clínicas e exames complementares (eletrocardiograma e radiografia de tórax) permitiram

determinar que todos estavam na fase crônica, sendo 9 na fase indeterminada e 5 apresentando cardiopatia (Tabela 1).

Isolamento e manutenção das cepas do T. cruzi no laboratório. Para o isolamento das cepa, o tubo digestivo dos triatomíneos positivos empregados no xenodiagnóstico foi homogeneizado com solução salina e inoculado intraperitonealmente em 3 camundongos albinos suíços não isogênicos, machos, pesando $10 \mathrm{a} 15 \mathrm{~g}$.

Tabela 1- Dados dos pacientes e virulência de 14 cepas do T. cruzi procedentes de três áreas de Minas Gerais.

\begin{tabular}{|c|c|c|c|c|c|c|c|c|c|}
\hline \multirow[b]{2}{*}{ Código da cepa } & \multicolumn{2}{|c|}{ Paciente } & \multirow[b]{2}{*}{ forma clínica } & \multirow{2}{*}{$\begin{array}{l}\text { PPP } \\
\text { (dias) }\end{array}$} & \multicolumn{2}{|c|}{ Pico de parasitemia* } & \multirow[b]{2}{*}{ inoculados } & \multicolumn{2}{|c|}{$\begin{array}{l}\text { Camundongos } \\
\text { mortos até } 60 \text { dias }(\mathrm{N})\end{array}$} \\
\hline & idade & sexo & & & $\mathrm{n}^{\circ}$ & dias & & $\mathrm{N} /$ vivos & $\%$ \\
\hline $\mathrm{P} 135-1$ & 71 & $M$ & indet & 5 & 157 & 28 & 10 & 0 & 0 \\
\hline P23-1 & 72 & M & card & 11 & 284 & 31 & 12 & $1 / 12$ & 8,3 \\
\hline $\lg 200$ & 66 & M & card & 8 & 619 & 20 & 10 & 0 & 0 \\
\hline $\lg 539$ & 72 & $\mathrm{~F}$ & indet & 5 & 2946 & 16 & 8 & 0 & 0 \\
\hline $\lg 62$ & 49 & $\mathrm{~F}$ & indet & 10 & 231 & 30 & 7 & 0 & 0 \\
\hline B1931 & 28 & M & indet & 10 & 1151 & 18 & 7 & 0 & 0 \\
\hline B2147 & 29 & $\mathrm{~F}$ & card & 5 & 3989 & 16 & 13 & 0 & 0 \\
\hline B615 & 42 & $\mathrm{~F}$ & indet & 5 & 1143 & 18 & 9 & $2 / 6$ & 33,3 \\
\hline B654 & 34 & $M$ & card & 6 & 274 & 16 & 12 & 0 & 0 \\
\hline B754 & 16 & $\mathrm{~F}$ & indet & 5 & 1447 & 24 & 12 & $1 / 12$ & 8,3 \\
\hline B768 & 15 & $\mathrm{~F}$ & indet & 6 & 274 & 16 & 10 & 0 & 0 \\
\hline B803 & 35 & $\mathrm{~F}$ & indet & 9 & 251 & 44 & 12 & 0 & 0 \\
\hline B84 & 21 & $\mathrm{~F}$ & card & 6 & 3089 & 30 & 9 & $1 / 7$ & 14,3 \\
\hline BE-25 & 27 & $\mathrm{~F}$ & indet & 12 & 337 & 20 & 8 & 0 & 0 \\
\hline
\end{tabular}

PPP: Período pre-patente; *: Média de parasitemia; P: Pains, Ig: Iguatama; B: Berilo; M: masculino;

F: Feminino; indet: indeterminada; card: cardíaca.

Foram feitas reinoculações em camundongos, utilizando tripomastigotas sanguíneos obtidos da fase aguda da infecção, em torno do pico da parasitemia para manutenção. O sangue foi obtido por punção do seio venoso orbital, usando pipeta Pasteur com heparina (Liquemine $\AA$, Roche) ou do coração usando seringas heparinizadas. Aproximadamente, $1 \times 10^{4}$ parasitos em $0,1 \mathrm{ml}$ foram inoculados por via intraperitoneal em cada camundongo.
Caracterização biológica. Estudo da parasitemia em camundongos. A partir de um grupo inicial de três camundongos infectados com as cepas em estudo, uma passagem foi efetuada para um segundo grupo de camundongos ( 7 a 13 animais). As inoculações foram feitas com $1 \times 10^{4}$ tripomastigotas sangüíneos, por via intraperitoneal. A pesquisa de formas tripomastigotas na corrente sangüínea dos camundongos foi feita diariamente a partir do $5^{\circ}$ dia até a observação de 
parasitos e, em seguida, a intervalos de 2 a 4 dias, com término de observação no $60^{\circ}$ dia após a inoculação.

A contagem dos tripomastigotas sangüíneos foi feita como descrito por Brener ${ }^{4}$. Foram observados 50 campos microscópicos de 400X. As curvas de parasitemia foram feitas utilizando a média aritmética das contagens de tripomastigotas sanguíneos nos camundongos sobreviventes em cada dia de contagem.

No presente estudo, considerou-se cepa de elevada parasitemia aquela que apresentava, picos médios maiores que 1500 parasitos por $5 \mu$; parasitemia média quando foram encontrados picos entre 500 e 1.499 tripomastigotas; e baixa parasitemia quando os picos foram inferiores a 500 formas sanguíneas.

Morfologia de tripomastigotas sangüíneos. A morfologia das formas parasitárias sangüíneas foi estabelecida em esfregaços de 2 em 2 dias durante a fase aguda da infecção. Um total de 2 a 5 esfregaços por animal foram feitos com sangue da cauda. Após secagem, fixação pelo metanol e coloração pelo Giemsa, as lâminas foram lidas em microscópio com aumento de 1000X. Foram procurados cinqüenta tripomastigotas por lâmina, ou lido todo o esfregaço nos casos de parasitemia baixa. Os critérios descritos por Brener e Chiari ${ }^{7}$ foram usados para se caracterizar as formas delgadas, largas e muito largas.
Virulência das cepas do T. cruzi. Para estudar a virulência das amostras considerou-se a infectividade, o período pré-patente (PPP), o curso da infecção, a curva de parasitemia e a mortalidade dos camundongos ${ }^{1816}$.

Histopatolologia. Dois a três animais, selecionados ao acaso, foram sacrificados usando vapores de éter, na fase aguda da infecção, geralmente entre os dias 15 e 30 após inoculação. Da mesma forma foi feito na fase crônica entre os dias 60 e 90 de infecção. Os órgãos colhidos durante a necropsia (cérebro, testículos, fígado, baço, rins, coração, esôfago, intestinos, bexiga e tríceps) foram colocados em recipientes contendo formalina $10 \%$ tamponada de Millong, clivados, incluídos em parafina e corados com hematoxilina-eosina.

Dois observadores examinaram cada corte em toda a sua extensão, determinando a presença do parasito (parasitismo tecidual) e de reação inflamatória. Utilizouse uma escala qualitativa para classificar o infiltrado inflamatório referente ao número de focos por campo microscópico de 100X: + um foco (discreto); ++ de 2 a 5 focos (moderado); +++ de 6 a 10 focos (intenso); ++++ mais de 10 focos (muito intenso).

As cepas foram classificadas segundo Andrade ${ }^{2}$ em três tipos biológicos ou biodemas: I, II ou III.

\section{RESULTADOS}

Três cepas (Ig539, B84 e B2147) apresentaram alta parasitemia, cujos picos ocorreram entre os dias 16 e 30 após a infecção. Cinco cepas (Ig200, B1931, B615, B654 e B754) induziram picos parasitêmicos médios irregulares ao longo do tempo de análise atingindo os níveis máximos em torno dos dias 16 e 24 após a infecção. Seis cepas (P135-1, P23-1, B803, Ig62, BE-25 e B768) se caracterizaram pela baixa parasitemia com os picos ocorrendo entre os dias 16 e 44 após a infecção (Tabela 1). $\mathrm{Na}$ maioria das cepas a parasitemia manteve-se baixa e patente até os 60 dias de observação.

Uma análise global revelou que as cepas das três áreas apresentaram PPP similares (de 5 a 12 dias). Entretanto, os maiores níveis de parasitemia foram observados nas amostras de Berilo. Individualmente, os PPP foram mais curtos nas cepas de alta (Ig539, B2147 e B84) e média (B1931, B654, B754, B615) parasitemia.

Ambas as cepas de Pains (P135-1 e P23-1) tiveram parasitemias baixas, sempre inferiores a $1 \times 10^{3}$ parasitos $/ 5 \mathrm{ml}$. Os PPP foram de 5 e 11 dias, ocorrendo os picos parasitêmicos em torno do $30^{\circ}$ dia de infecção. Após 50 dias de análise, já não era mais possível encontrar tripomastigotas no sangue dos animais.

As cepas provenientes de Iguatama apresentaram parasitemias diferentes. O PPP variou de 5 a 10 dias, sendo menor (5 dias) na cepa Ig539 de elevada parasitemia e maior em Ig62 de baixa parasitemia. Os picos parasitêmicos aconteceram, em média em torno do dia 22, variando entre os dias 16 e 32 segundo a cepa.

Em Berilo também foi observada variabilidade dos níveis parasitêmicos de uma cepa para outra. Quatro cepas (B754, B654, B615 e B1931) tiveram níveis médios, duas (B84 e B2147) apresentaram alta parasitemia, e três (B768, BE-25 e B803) se caracterizaram como de baixa parasitemia. Os picos de parasitemia ocorreram entre os dias 16 e 44, com uma média em torno do dia 22. As cepas de Berilo apresentaram PPP menores quando comparado com as outras regiões, porém também variando de 5 a 12 dias.

Mortalidade. Apenas quatro cepas (B84, B615, B754 P23-1) foram capazes de produzir mortalidade nos camundongos infectados. Não houve mortalidade em dez casos (P135-1, Ig200, Ig539, lg62, B768, B803, B654, B1931, B2147 e BE-25). A mortalidade geral oscilou entre 0 e $33,3 \%$. A morte dos animais ocorreu entre os dias 20 e 30 nas cepas B84 e B754, coincidindo com os níveis elevados de parasitemia. Nas cepas P231 e B615 a morte ocorreu mais tardiamente por ocasião da queda dos níveis parasitêmicos (dias 39 e 50 respectivamente).

Morfologia de tripomastigotas sanguíneos. Neste segmento do estudo, foram analisadas apenas 8 cepas, 5 com parasitemia média (Ig200, B654, B1931, B754, B615) e 3 com alta (Ig539, B84, B2147), uma vez que 6 apresentaram baixa parasitemia impedindo a análise morfológica. Em todas as cepas predominaram as 
formas largas durante o curso da infecção. Nas cepas B2147, B84 e lg539 de alta parasitemia, observou-se a presença de formas delgadas, em baixa percentagem, entre os dias 11 e 19, que desapreciam em torno do dia 20 de infecção. Nas cepas B754, Ig200, B1931 e B654 de média parasitemia, também foram observadas formas delgadas porém em percentagens não significativas.

Histopatologia. Parasitismo tecidual foi observado durante a fase aguda em 64,3\% (9/14) dos casos
(Tabela 2), sendo os órgãos mais acometidos em ordem de freqüência: coração $(64,3 \%)$, músculo esquelético $(28,6 \%)$, bexiga $(14,3 \%)$ e fígado $(7,1 \%)$. Todos os camundongos infectados com cepas de média (3/3) e alta parasitemia (5/5) apresentaram parasitismo tecidual e inflamação na fase aguda. Somente uma cepa (B803) de baixa parasitemia $(1 / 6-16,6 \%)$ induziu o aparecimento de parasitos em três órgãos distintos (coração, músculo esquelético e músculo liso da bexiga) durante essa fase.

Tabela 2 - Achados histopatológicos em coração, músculo esquelético e bexiga de camundongos infectados com 14 cepas do T. cruzi isoladas de pacientes chagásicos crônicos de três áreas de Minas Gerais.

\begin{tabular}{|c|c|c|c|c|c|c|c|}
\hline \multirow[b]{2}{*}{ Cepa } & \multirow[b]{2}{*}{ Achado histopatolog } & \multicolumn{6}{|c|}{$\begin{array}{l}\text { Órgão } \\
\text { músculo }\end{array}$} \\
\hline & & f. a. & f. c. & f. a. & f. c. & f. a. & f. c. \\
\hline \multirow[t]{2}{*}{ P135-1 } & parasitos & - & - & - & - & - & - \\
\hline & inflamação & + & + & + & + & - & + \\
\hline \multirow[t]{2}{*}{ P23-1 } & parasitos & - & - & - & - & - & - \\
\hline & inflamação & + & + & - & - & - & - \\
\hline \multirow[t]{2}{*}{$\lg 200$} & parasitos & $x$ & - & - & - & - & - \\
\hline & inflamação & ++ & + & ++ & + & + & + \\
\hline \multirow[t]{2}{*}{$\lg 539$} & parasitos & $x$ & - & - & - & - & - \\
\hline & inflamação & ++ & ++ & + & - & & ++ \\
\hline \multirow[t]{2}{*}{$\lg 62$} & parasitos & - & - & - & - & - & - \\
\hline & inflamação & + & + & + & + & - & - \\
\hline \multirow[t]{2}{*}{ B1931 } & parasitos & $x$ & - & $x$ & - & - & - \\
\hline & inflamação & +++ & - & +++ & - & +++ & - \\
\hline \multirow[t]{2}{*}{ B2147 } & parasitos & $x$ & $x$ & $x$ & - & $x$ & - \\
\hline & inflamação & +++ & ++ & ++ & + & + & - \\
\hline \multirow[t]{2}{*}{ B615 } & parasitos & $x$ & - & - & - & - & - \\
\hline & inflamação & + & + & + & + & + & + \\
\hline \multirow[t]{2}{*}{ B654 } & parasitos & $x$ & - & - & - & - & - \\
\hline & inflamação & +++ & ++ & ++ & + & - & - \\
\hline \multirow[t]{2}{*}{ B754 } & parasitos & $x$ & - & - & - & - & - \\
\hline & inflamação & ++ & ++ & ++ & + & ++ & + \\
\hline \multirow[t]{2}{*}{ B768 } & parasitos & - & - & - & - & - & - \\
\hline & inflamação & + & + & + & - & - & - \\
\hline \multirow[t]{2}{*}{ B803 } & parasitos & $x$ & - & $x$ & - & $x$ & - \\
\hline & inflamação & ++ & ++ & + & + & + & ++ \\
\hline \multirow[t]{2}{*}{ B84 } & parasitos & $x$ & - & $x$ & $x$ & - & - \\
\hline & inflamação & ++ & + & - & - & - & ++ \\
\hline \multirow[t]{2}{*}{ BE-25 } & parasitos & - & - & - & - & - & - \\
\hline & inflamação & ++ & + & ++ & + & + & + \\
\hline
\end{tabular}

f.a.: fase aguda; f.c.: fase crônica; X: presencia de parasitos; -: ausência de parasitos ou inflamação.

$\mathrm{Na}$ fase crônica, o parasitismo tecidual foi encontrado apenas em camundongos infectados com 2 cepas isoladas de pacientes de Berilo (B2147 e B84) sendo ambas de alta parasitemia (2/14 - 14,3\%). A presença de ninhos de amastigotas foi verificada no coração e músculo esquelético, respectivamente. Focos inflamatórios discretos a moderados (+/4 ou ++/ 4) foram observados em todas os casos na fase crônica (Tabela 2), sendo os órgãos mais atingidos coração (92,9\%), fígado (92,9\%), músculo esquelético $(64,3 \%)$ e bexiga $(57,1 \%)$.
As cepas B84 e B2147 que determinaram parasitismo tissular na fase crônica, cursaram também com presença de $T$. cruzi tecidual na fase aguda e alta parasitemia.

Não foram observadas formas amastigotas nos intestinos dos animais sacrificados.

Relacionando a área de procedência das cepas, sua parasitemia e as lesões histopatológicas nos camundongos, pode-se constatar maior parasitismo tecidual nas cepas de Berilo com média a alta parasitemia, acometendo principalmente o miocárdio 
embora também tenham atingido músculo esquelético, bexiga e fígado. Cepas de alta e média parasitemia de Iguatama acometeram miocárdio. Em nenhum dos camundongos infectados pelas duas cepas isoladas em Pains foram observadas formas amastigotas. Contudo, deve-se considerar que a maioria das cepas (9) foi isolada de pacientes de Berilo.

Biodema das cepas do T. cruzi. Embora os dados não sejam suficientes para enquadrar todas as cepas num biodema específico ${ }^{1}$, pode-se sugerir a classificação da Tabela 3. Em Pains ambas as cepas foram caracterizadas como pertencentes ao biodema III. Em Iguatama 2 cepas são do biodema II e 1 do III; enquanto que em Berilo 7 cepas apresentaram um comportamento similar ao biodema II, e 2 ao do biodema III. Nas Figuras 1 e 2 apresentam-se os perfis parasitêmicos das cepas classificadas como biodema II e III, respectivamente.

Tabela 3 - Relação entre os dados experimentais e clínicos das 14 cepas do T. cruzi isoladas de pacientes chagásicos crônicos de três áreas de Minas Gerais.

\begin{tabular}{|c|c|c|c|c|c|c|c|c|}
\hline \multirow[b]{2}{*}{ Cepa do T. cruzi } & \multicolumn{2}{|c|}{ Virulência } & \multicolumn{2}{|c|}{ Histopatologia/fase aguda } & \multirow[b]{2}{*}{ Biodema da cepa } & \multicolumn{3}{|c|}{ Paciente } \\
\hline & parasitemia & mortalidade (\%) & parasito & órgãos acometidos $\left(\mathrm{n}^{\circ}\right)$ & & idade (anos) & sexo & forma clínica \\
\hline P135-1 & baixa & 0 & - & 0 & III & 71 & M & indet \\
\hline P23-1 & baixa & 8,3 & - & 0 & III & 72 & M & card \\
\hline $\lg 200$ & média & 0 & + & 1 & II & 66 & $M$ & card \\
\hline $\lg 539$ & alta & 0 & + & 1 & II & 72 & $\mathrm{~F}$ & indet \\
\hline $\lg 62$ & baixa & 0 & - & 0 & III & 49 & $\mathrm{~F}$ & indet \\
\hline B1931 & média & 0 & + & 1 & II & 28 & $M$ & indet \\
\hline B2147 & alta & 0 & + & 3 & II & 29 & $\mathrm{~F}$ & card \\
\hline B615 & média & 33,3 & + & 1 & II & 42 & $\mathrm{~F}$ & indet \\
\hline B654 & média & 0 & + & 1 & II & 34 & M & card \\
\hline B754 & média & 8,3 & + & 1 & II & 16 & $\mathrm{~F}$ & indet \\
\hline B768 & baixa & 0 & - & 0 & II & 15 & $\mathrm{~F}$ & indet \\
\hline B803 & baixa & 0 & + & 3 & III & 35 & $\mathrm{~F}$ & indet \\
\hline B84 & alta & 14,3 & + & 1 & III & 21 & $\mathrm{~F}$ & card \\
\hline BE-25 & baixa & 0 & - & 0 & II & 27 & $\mathrm{~F}$ & indet \\
\hline
\end{tabular}

M: masculino; F: Feminino; indet: indeterminada; card: cardíaca.

A

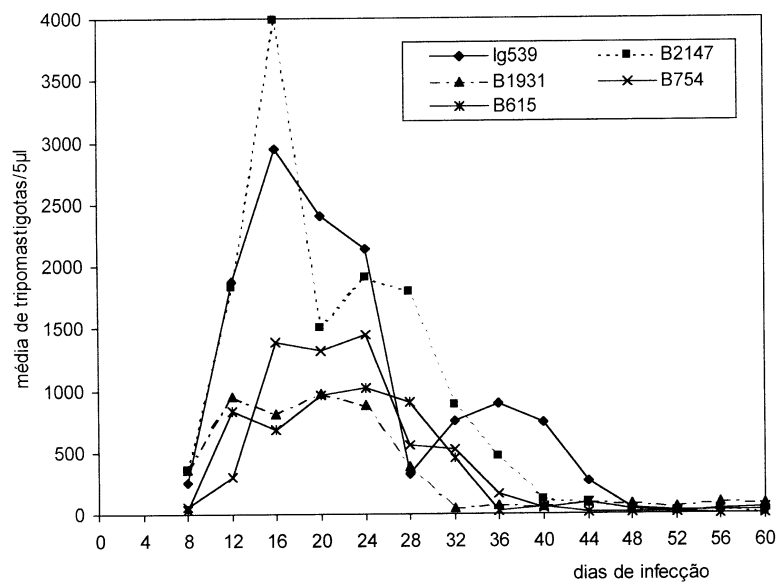

B

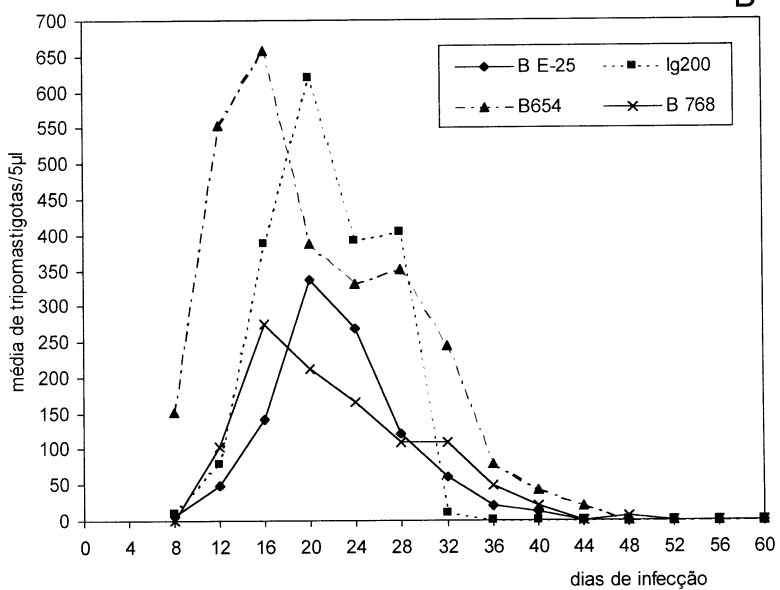

Figura 1. Parasitemia em camundongos suíços infectados por cepas do T. cruzi classificadas dentro do biodema II. A: Cepas Ig539, B2147, B1931, B754 e B615. B: Cepas lg200, B654, B768 e BE-25.

Relações clínico-experimentais. Ao se relacionar a forma clínica dos pacientes com a virulência e patogenicidade das cepas, observou-se que das 6 cepas de maior patogenicidade, 3 foram isoladas de pacientes na fase indeterminada e 3 isoladas de pacientes na fase crônica cardíaca. Em 2 (B2147 e B84) das 14 cepas testadas, houve correlação entre a virulênica e patogenicidade nos camundongos (elevada parasitemia e alta mortalidade) e a alta morbidade (forma cardíaca) apresentada pelos pacientes das quais foram isoladas.

Quando considerado apenas a parasitemia nos camundongos infectados e a forma clínica, pode ser observada uma relação não comprovada estatisticamente. 


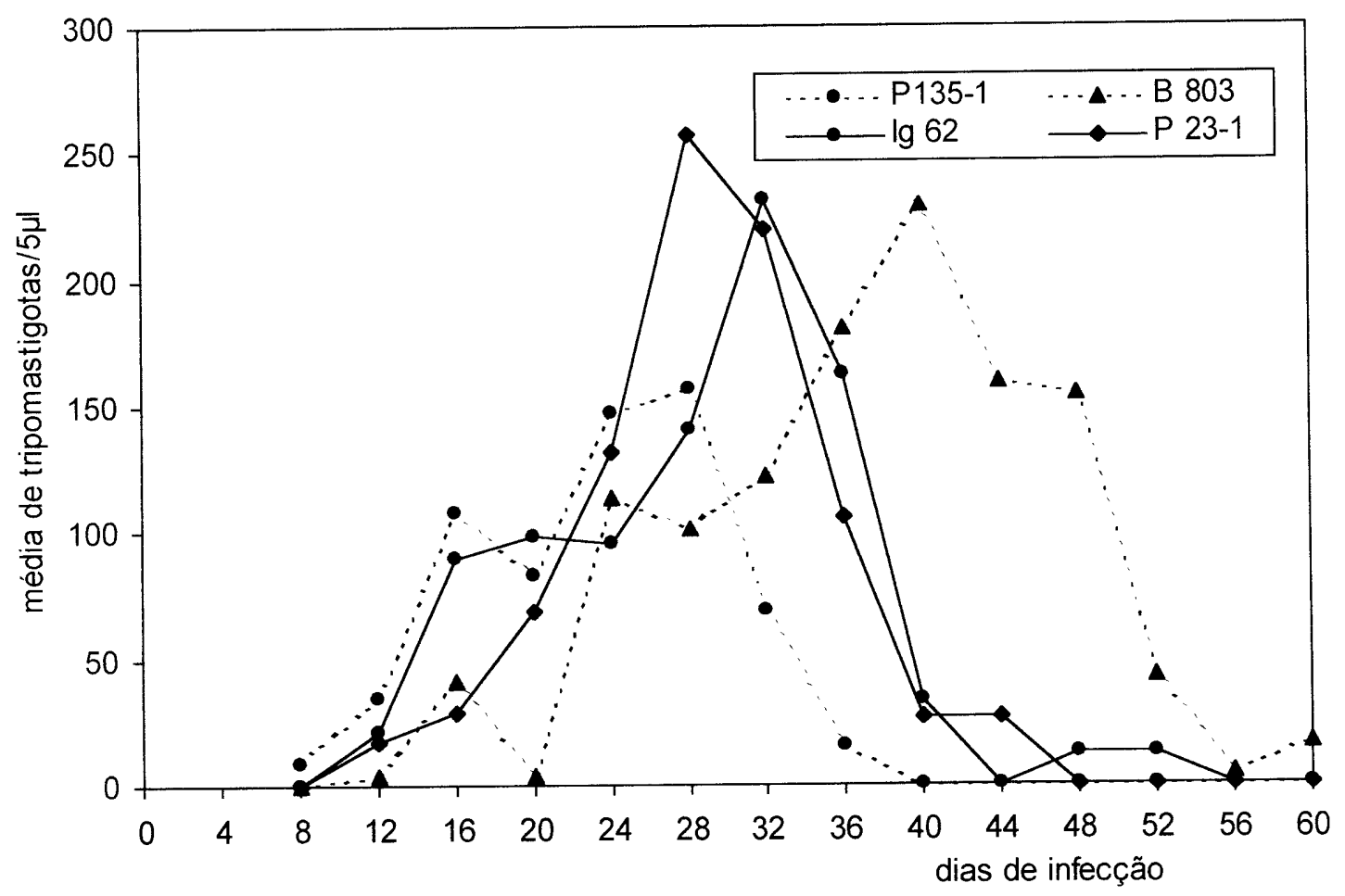

Figura 2 - Parasitemia em camundongos suiços infectados por cepas do T. cruzi classificadas dentro do biodema III.

As cepas dos pacientes com a forma cardíaca foram as que determinaram parasitemias médias ou elevadas nos camundongos. Todas as cepas que apresentavam níveis parasitêmicos baixos no modelo murino, procederam de pacientes na fase indeterminada.
Das 9 cepas do biodema II, 3 foram isoladas de pacientes portadores da forma cardíaca e 6 de pacientes com forma indeterminada. Três das 5 cepas classificadas como biodema III, procederam de pacientes com forma indeterminada (Tabela 3).

\section{DISCUSSÃO}

Em geral, o comportamento biológico das cepas provenientes de pacientes moradores em 3 distintos municípios de Minas Gerais apresentaram o seguinte comportamento biológico no camundongo suiço: 1) períodos pré-patentes variáveis de 5 até 12 dias, sendo mais curtos nas cepas de maior parasitemia; 2) multiplicação relativamente lenta com picos de parasitemia entre os dias 16 e 30;3) presença de cepas de alta, média e baixa parasitemia, sendo a maioria, de média e baixa; 4) poucas formas delgadas no início da infecção com predomínio de formas largas; 5) mortalidade acumulada baixa, variando de 0 a 33,3\%; 6) baixa virulência, porém variável de uma cepa para outra e entre as diferentes áreas; 7) miocardiotropismo.

Em relação à parasitemia, o perfil observado foi similar ao obtido com outras cepas do $T$. cruzi recémisoladas de pacientes chagásicos crônicos ${ }^{13} 16$.

A maioria das cepas não causou morte dos animais durante 60 dias de observação, e naqueles onde ocorreu, as taxas de mortalidade foram baixas. Portanto, baseados na parasitemia e mortalidade, as cepas podem ser classificadas como de baixa virulência. Estudos prévios têm mostrado que a maioria das cepas isoladas de humanos com DC crônica e inoculadas em camundongos é de baixa virulência, geralmente com níveis parasitêmicos baixos ou sub-microscópicos ${ }^{18912131416}$.

Em todas as amostras estudadas as formas largas foram as mais freqüentemente observadas durante a fase aguda da infecção, achado comum em cepas de baixa virulência ${ }^{21013}$. Uma percentagem variável, porém sempre baixa, de formas delgadas foi evidenciada por algumas vezes no início da infecção. As cepas de maior parasitemia foram as que apresentaram maior percentual de formas delgadas em relação às outras cepas, coincidindo com os resultados de Andrade ${ }^{1}$, Carneiro et $\mathrm{al}^{15}$ e Schlemper $\mathrm{Jr}^{16}$.

$\mathrm{Na}$ maioria das cepas a presença de parasitismo tecidual foi coincidente com a maior parasitemia da cepa nos camundongos. Em $63,4 \%$ dos casos, houve comprometimento do miocárdio e, em $28,6 \%$ dos casos, do músculo esquelético. Esses resultados coincidem com aqueles de Schlemper $\mathrm{Jr}^{16}$ que estudando cepas 
procedentes de Iguatama e Virgem da Lapa, um município vizinho a Berilo, verificou que as 7 cepas apresentavam miocardiotropismo e a maior intensidade do parasitismo foi observada em cepas de elevada parasitemia.

Com a exceção de 1 caso (cepa B803), nas cepas que cursaram com baixas parasitemias não foram vistos parasitos nos tecidos, e as lesões inflamatórias foram de baixa intensidade, concordando com os resultados obtidos por Andrade ${ }^{2}$ e Schlemper Jr et al ${ }^{17}$.

A evolução, picos da parasitemia e a baixa mortalidade permitiu incluir algumas das cepas estudadas no biodema III (Figura 2), porém sem demonstrar o acometimento preferencial da musculatura esquelética, característico das cepas deste biodema. Outras cepas preencheram as características do biodema II (Figura 1), tendo principalmente a seu favor o perfil parasitêmico e o miocardiotropismo. Oliveira et $\mathrm{al}^{13}$ também enfrentaram dificuldades em enquadrar sua cepa em um determinado padrão biológico.

Os resultados mostram uma heterogeneidade das cepas, devido à presença de dois biodemas em 2 das 3 áreas estudadas. O biodema II foi o mais freqüente tanto em Berilo como em Iguatama, sendo que as duas cepas procedentes de Pains eram do biodema III. No estudo realizado por Schlemper $\mathrm{Jr}^{16}$, as cepas do $T$. cruzi provenientes de Iguatama apresentaram um padrão do tipo II.

A heterogeneidade de biodemas presentes nesta e outras regiões de Minas Gerais descrita na literatura ${ }^{38}$ 16 , respalda os resultados do presente estudo que evidenciou a presença simultânea de ambos os biodemas. Este mesmo fenômeno foi descrito por Andrade e Magalhães ${ }^{3}$ que determinaram a presença simultânea dos biodemas II e III em Montalvania (norte de Minas Gerais) e por Carneiro et al ${ }^{8}$ em Bambuí (oeste de Minas Gerais). A pesar de esta heterogeneidade, os biodemas geralmente têm uma distribuição ubíqua, porém, sempre com uma tendência ao predomínio de um tipo em uma mesma área ${ }^{3}$.

Geralmente a transferência dos dados experimentais para a interpretação da doença humana é difícil ${ }^{1} 38131416$. Isto ficou também demonstrado no presente estudo, pois não houve relação entre os dados no animal experimental e as formas clínicas dos pacientes. A falta de uma relação bem definida entre os níveis parasitêmicos e as lesões histopatológicas no modelo experimental, com a forma clínica da doença sustenta a hipótese de que a patogenia da DC não se deve a um único fator.

Quando se tenta relacionar o biodema da cepa com a gravidade da forma clínica do paciente também não foi possível se evidenciar qualquer relação. Embora fosse visto que o biodema III se relacionasse mais com pacientes com forma indeterminada, deve-se salientar que 9 dos 14 pacientes apresentavam essa forma, portanto pode ser um achado apenas casual.

Em conclusão das 14 cepas estudadas, 9 foram clasificadas como pertencentes ao biodema II e 5 ao III. Foi demonstrada a presença simultanea dos dois biodemas em Berilo e Iguatama. Não foi verificada uma relação entre a DC experimental e humana nas areas estudadas.

\section{AGRADECIMENTOS}

Registramos nossos agradecimentos aos Drs. José Borges Pereira, Márcio Neves Bóia e Naurea dos Santos por sua participação no inquérito sorológico e clínico-epidemiológico. A José de Souza Nogueira, Pauzanias Fernandes e Maria Celeste Spata pela assistência técnica.

\section{REFERÊNCIAS BIBLIOGRÁFICAS}

1. Andrade SG. Caracterização de cepas de Trypanosoma cruzi isoladas do Recôncavo Baiano: Contribuição ao estudo da patologia geral da doença de Chagas em nosso meio. Revista de Patologia Tropical 3:65-121, 1974.

2. Andrade SG. Morphological and behavioural characterization of Trypanosoma cruzi strains. Revista da Sociedade Brasileira de Medicina Tropical 18 (supl I.):39-46, 1985.

3. Andrade SG, Magalhães JB. Biodemes and zymodemes of Trypanosoma cruzi strains: correlations with clinical data and experimental pathology. Revista da Sociedade Brasileira de Medicina Tropical 30:27-35, 1997.

4. Brener Z. Therapeutic activity and criterion of cure on mice experimentally infected with Trypanosoma cruzi. Revista do Instituto de Medicina Tropical de São Paulo 4:398-396, 1962.

5. Brener Z. The behavior of slender and stout forms of Trypanosoma cruzi in the blood stream of normal and immune mice. Annals of Tropical Medicine and Parasitology 63:215-220, 1969.
6. Brener Z. Intraspecific variation in Trypanosoma cruzi: two types of parasite populations resenting distinct characteristics. Pan-American Health Organization, Scientific Publication 347:11-21, 1977.

7. Brener Z, Chiari E. Variações morfológicas observadas em diferentes amostras de Trypanosoma cruzi. Revista do Instituto de Medicina Tropical de São Paulo 5:220-224, 1963.

8. Carneiro M, Romanha AJ, Chiari E. Biological characterization of Trypanosoma cruzi strains from different zymodemes and schizodemes. Memórias do Instituto Oswaldo Cruz 86:387-393, 1991.

9. Fernandes CD, Murta SMF, Cerávolo IP, Krug LP, Vidigal PG, Steindel M, Nardi N, Romanha AJ. Characterization of Trypanosoma cruzi isolated from chronic chagasic patients, triatomines and opossums naturally infected from the State of Rio Grande do Sul, Brazil. Memórias do Instituto Oswaldo Cruz 92:343-351, 1997.

10. Miles MA, Toye PJ, Oswald SC, Godfrey DG. The identification by isoenzyme patterns of two distinct strain-groups of Trypanosoma cruzi circulating independently in a rural area of 
Brazil. Transactions of the Royal Society of Tropical Medicine Hygiene 71:217-225, 1977.

11. Morel C, Chiari E, Camargo EP, Mattei DM, Romanha AJ, Simpson L. Strains and clones of Trypanosoma cruzi can be characterized by pattern of restriction endonuclease products of kinetoplast DNA minicircles. Proceeding of National Academy of Sciences of USA 77:6810-6814, 1980.

12. Oliveira EC, Stefani MMA, Campos DE, Andrade ALSS, Silva SA, Rasi A, Luquetti AO. Trypanosoma cruzi stocks isolated from acute Chagas disease patient lead to lethal murine infection. Transactions of the Royal Society of Tropical Medicine and Hygiene 91:25-27, 1997.

13. Oliveira EC, Stefani MMA, Luquetti AO, Vêncio EF, Moreira MAR, Souza C, Rezende JM. Trypanosoma cruzi and experimental Chagas' disease: characterization of a stock isolated from a patient with associated digestive and cardiac form. Revista da Sociedade Brasileira de Medicina Tropical 26:25-33, 1993.

14. Petana WB, Coura JR. Experimental studies on Trypanosoma (Schizotrypanum) cruzi strains isolated from man, animals and from triatomines bugs in Brazil. Revista da Sociedade Brasileira de Medicina Tropical 8:315-323, 1974.

15. Prata A. Significance of Trypanosoma cruzi differentiation and selection, relationship with clinical and epidemiological varieties.
Revista da Sociedade Brasileira de Medicina Tropical 18(supl I):9-16, 1985.

16. Schlemper Jr BR. Caracterização de cepas do Trypanosoma cruzi isoladas de pacientes com diferentes formas clínicas da doença de Chagas. Tese de Doutorado, Universidade Federal de Rio de Janeiro, Rio de Janeiro, 1982.

17. Schlemper Jr BR, Avila CM, Coura JR, Brenner Z. Course of infection and histopathological lesions in mice infected with seventeen Trypanosoma cruzi strains isolated from chronic patients. Revista da Sociedade Brasileira de Medicina Tropical 16:23-30, 1983.

18. Steindel M, Dias Neto E, Menezes C, Romanha A, Simpson AJG. Random amplified polymorphic DNA analysis of Trypanosoma cruzi strains. Molecular and Biochemical Parasitology 60:71-80, 1983.

19. Tibayrenc M, Ayala F. Isozyme variability in Trypanosoma cruzi, the agent of Chagas' disease: genetical, taxonomical and epidemiological significance. Evolution 42:277-292, 1988.

20. Tibayrenc M, Brenière SF. Trypanosoma cruzi: major clones rather than principal zymodemes. Memórias do Instituto Oswaldo Cruz 83:249-255, 1988.

21. World Health Organization. Control of Chagas Disease. Report of a WHO Expert Committee, WHO Technical Report Series no 811, Geneva, 1991. 